\title{
The modulatory influence of the functional COMTVal158Met polymorphism on lexical decisions and semantic priming
}

\author{
Martin Reuter ${ }^{1}{ }^{*}$, Christian Montag $^{1}$, Kristina Peters ${ }^{2}$, Anne Kocher ${ }^{1}$ and Markus Kiefer ${ }^{3}$ \\ Department of Psychology, University of Bonn, Bonn, Germany \\ 2 Department of Psychology, University of Giessen, Giessen, Germany \\ ${ }^{3}$ Department of Psychiatry, University of UIm, UIm, Germany
}

\section{Edited by:}

Silvia A. Bunge, University of California

Berkeley, USA

Reviewed by:

Chris Blais, University of California Berkeley, USA

Adam Green, Yale University, USA

${ }^{*}$ Correspondence:

Martin Reuter, Laboratory of

Neurogenetics, Department of

Psychology, University of Bonn,

Kaiser-Karl-Ring 9, D-53111 Bonn,

Germany.

e-mail:martin.reuter@uni-bonn-diff.de
The role of the prefrontal Cortex (PFC) in higher cognitive functions - including working memory, conflict resolution, set shifting and semantic processing - has been demonstrated unequivocally. Despite the great heterogeneity among tasks measuring these phenotypes, due in part to the different cognitive sub-processes implied and the specificity of the stimulus material used, there is agreement that all of these tasks recruit an executive control system located in the PFC. On a biochemical level it is known that the dopaminergic system plays an important role in executive control functions. Evidence comes from molecular genetics relating the functional COMT Val158Met polymorphism to working memory and set shifting. In order determine whether this pattern of findings generalises to linguistic and semantic processing, we investigated the effects of the COMTVal158Met polymorphism in lexical decision making using masked and unmasked versions of the semantic priming paradigm on $N=104$ healthy subjects. Although we observed strong priming effects in all conditions (masked priming, unmasked priming with short/long stimulus asynchronies (SOAs), direct and indirect priming), COMT was not significantly related to priming, suggesting no reliable influence on semantic processing. However, COMTVal158Met was strongly associated with lexical decision latencies in all priming conditions if considered separately, explaining between 9 and $14.5 \%$ of the variance. Therefore, the findings indicate that COMT mainly influences more general executive control functions in the PFC supporting the speed of lexical decisions.

Keywords: semantic priming, executive control, catechol-O-methyltransferase, COMT Val158Met polymorphism, prefrontal cortex

\section{INTRODUCTION}

Numerous neurophysiological and imaging studies have demonstrated the prominent role of the prefrontal cortex (PFC) for a variety of cognitive functions (e.g. D'Esposito et al., 2000; Duncan and Owen, 2000; Liu et al., 2008; Reuter et al., 2008; Volle et al., 2008). Although these cognitive functions show a large diversity with respect to the cognitive sub-processes involved there is agreement that the common denominator is an executive control system assumed to be located in the PFC (Kiefer et al., 1998; Posner and DiGirolamo, 1998; Faw, 2003). The executive control system organizes cognition and behavior when routines are not available or are ineffective for task performance (Kiefer et al., 2005). The executive control system plays an important role in a variety of tasks, such as manipulation of information in working memory (e.g., operation span tasks), conflict resolution (e.g. the Stroop interferences task), set shifting (e.g. the Wisconsin Card Sorting Test, WCST) and stimulus classification (Duncan, 2001). In addition to classical executive functions, PFC also contributes to various aspects of controlled word processing and word recognition. In particular, left inferior PFC (LIPFC) has been found to be involved in semantic memory retrieval supporting use generation, semantic classification, and semantic priming (Wagner et al., 2001; Kiefer et al., 2007). It has been proposed that LIPFC guides retrieval of semantic information by facilitating semantic access to aspects related to the context (Thompson-Schill, 2003; Kiefer et al., 2005). Results from imaging studies also stress the importance of LIPFC in retrieval of phonological word information (Poldrack et al., 1999). Presumably, semantic and phonological retrieval is supported by different sub-regions of LIPC (Poldrack et al., 1999; Thompson-Schill, 2003). Correspondingly, visual word recognition within a lexical decision task, a task that involves word retrieval from long-term memory as well as phonological and semantic processing (Neely, 1991; Kiefer et al., 2008), highly depends on neural circuits in LIPFC (Edwards et al., 2005). The functional significance of LIPFC for the lexical decision task is reflected by increased activity in this region as a function of difficulty when classifying words and pseudowords (Liu et al., 2004; Edwards et al., 2005). In contrast, the dorsolateral prefrontal cortex (DLPFC) seems to be crucial for non-semantic executive control tasks like the WCST (e.g. Monchi et al., 2001).

Although the neurobiological basis of cognitive functions has been investigated intensively by means of EEG and imaging studies yielding converging evidence for the importance of the PFC, the neurochemical underpinnings are less understood. The first hints for the hypothesis that the neurotransmitter dopamine (DA) is a crucial element for executive control came from studies in schizophrenic patients (Callaway, 1970). More recently, pharmacological challenge studies using dopaminergic drugs such as L-Dopa (e.g. 
Kischka et al., 1996; Vijayraghavan et al., 2007), in addition to genetic studies (e.g. Egan et al., 2001; Goldberg et al., 2003; Mattay et al., 2003; Reuter et al., 2005b; Bertolino et al., 2006), suggest that the cognitive processes involved in executive function are strongly influenced by the dopaminergic system. Under physiological conditions, cognitive performance is positively related to the amount of DA in the PFC. Besides receptor sensitivity and amount of DA release the degree of DA catabolism is essential for extra cellular DA levels. DA degradation in the PFC is under the control of the enzyme catechol-O-methyltransferase (COMT). For instance, a functional single nucleotide polymorphism (SNP) in the COMT gene, Val158Met, leading to a 3- to 4-fold difference in the enzyme activity by means of an amino acid exchange from valine (Val) to methionine (Met) in codon 158 (Lachman et al., 1996) has been related to cognitive functioning in numerous studies (Winterer and Goldman, 2003; Goldberg and Weinberger, 2004; Tunbridge et al., 2006). High DA catabolism (ValVal genotype) results in low DA concentration in the PFC and vice versa for low DA catabolism (MetMet genotype), whilst intermediate DA levels are characteristic for the heterozygous ValMet genotype. Converging evidence across numerous studies demonstrating influence on DA catabolism has highlighted COMT Val158Met as a critical genetic marker in the cognitive neuroscience literature. The most frequently studied phenotypes in this context are working memory and executive control assessed by the Wisconsin Card Sorting Test (Tunbridge et al., 2006; Barnett et al., 2007). Conversely, although semantic processing depends on PFC function and is presumably also modulated by DA activity (see below), there have been few molecular genetic studies on linguistic processing and none on semantic priming. Therefore, the present study is the first to investigate whether the COMT Val158Met polymorphism can account for individual differences in lexical decision-making within semantic priming paradigms.

Semantic priming generally refers to the facilitation of a response to a target stimulus that is preceded by a semantically related prime (Neely, 1977). For instance, in a lexical decision task (word/pseudoword decision), responses to a target word are faster, when it is preceded by a semantically related prime word. In support for the hypothesis that lexical-semantic processing depends on prefrontal DA activity, patients with schizophrenia showed increased semantic priming for directly- (hen-egg) and indirectly-(lemon [sour]-sweet) related prime-target word pairs, compared to healthy control subjects (Manschreck et al., 1988; Spitzer et al., 1994; Maher et al., 1996; Weisbrod et al., 1998). Priming in schizophrenic patients increased particularly at short stimulus onset asynchronies (SOAs) of $200 \mathrm{~ms}$ for indirectly related prime-target word pairs (Spitzer et al., 1993). Control subjects, in contrast, usually showed under this experimental condition little or no indirect priming (de Groot, 1983; Balota and Lorch, 1986; Spitzer et al., 1993; Kiefer et al., 1998). Because increased direct and indirect priming in patients with schizophrenia was observed at short SOAs, at which strategic priming processes are less effectively used (Neely, 1977, 1991), it is assumed that schizophrenia patients exhibit exaggerated automatic priming processes (Kiefer et al., 2005), putatively caused by low prefrontal DA activity. In support of this view, schizophrenia patients suffer from low prefrontal dopamine activity (e.g., Dolan et al., 1995). Furthermore, administration of L-dopa, a precursor of dopamine, has been found to decrease indirect priming in healthy subjects compared to a placebo control group, which showed strong indirect priming at the selected long SOA of $700 \mathrm{~ms}$ (Kischka et al., 1996). Finally, in a behavioural study investigating individual differences in working memory capacity, executive functions and semantic priming within the healthy population, Kiefer et al. (2005) found increased priming effects in individuals with low working memory capacity and inferior performance in the Stroop task probing executive functions suggesting a common neural correlate in the PFC. Hence, there are several lines of evidence for a modulatory role of DA in semantic processing. It is proposed that a high level of DA activity decreases the amount of semantic priming by focusing spreading activation during lexical access to the concept denoted by a given word (e.g., the prime word), thereby reducing the influence of the prime (Kiefer et al., 2005). This is potentially because semantic priming depends on an optimal signal-to-noise ratio triggered by DA in concert with GABA and glutamate transmission which is disturbed during psychosis (Winterer and Weinberger, 2004).

The relationship between DA levels and performance in cognitive tasks is far from being simple: Molecular genetic studies suggest a linear relationship reporting an allele load effect for the COMT Val 158Met polymorphism while pharmacological studies point to an inverted U shape function. In genetic studies, carriers of the Met/ Met genotype showed the best, carriers of the Val/Val allele the worst and subjects with the heterozygous Val/Met genotype intermediate performance (Goldberg and Weinberger, 2004). It is argued that the number of met alleles is positively related to prefrontal DA levels caused by a linear decrease in the catabolic enzyme activity with the number of Met alleles (Tunbridge et al., 2006). Interestingly, Mattay et al. (2003) reported better performance in carriers of the Val allele (and a decline in the Met/Met group) in a working memory task after a pharmacological challenge with the DA-agonist amphetamine. The decline in performance in the Met/Met group after amphetamine intake suggests that the association between performance and DA levels has not a linear, but fitted best by an inverted ' $U$ ' function, i.e. activation of the DA system by working memory load and amphetamine pushes these subjects beyond their optimal activation level. Nevertheless, there is mounting evidence that under physiological conditions, i.e. without a pharmacological manipulation, carriers of the Met/Met genotype exhibit superior performance in cognitive tasks. While the influence of the COMT Val 158Met polymorphism on executive function has meanwhile been well documented, it is unknown whether DA modulation of prefrontal functions beyond classical executive control, such as word recognition and semantic priming, are mediated by the same polymorphism.

In the present study, we investigated the effect of the COMT Val 158Met polymorphism on lexical decision-making within a semantic priming paradigm. As outlined above, the lexical decision task requires visual word recognition and involves controlled retrieval of lexical, phonological and semantic word information (e.g., Neely, 1991). Neuroimaging studies have shown that lexical decisions highly depend on areas in left inferior prefrontal cortex (Edwards et al., 2005). The lexical decision task is therefore a good candidate to study the influence of the COMT Val 158Met 
polymorphism on controlled processing outside the classical area of executive tasks. In order to assess semantic processing in detail, we used a masked and an unmasked priming paradigm. In the masked priming procedure (e.g., Kiefer, 2002; Kiefer and Brendel, 2006), conscious perception of the prime is eliminated by displaying a pattern mask (i.e., a random sequence of letters) before and after the prime. In addition, we also administered an unmasked version of the priming paradigm, in which the semantic distance between prime and target was systematically varied by including directly related, indirectly related and non-related prime-target pairings. While masked priming specifically reflects automatic priming processes, unmasked priming with visible stimuli additionally depends on controlled priming processes (Posner and Snyder, 1975; Neely, 1977; Kiefer, 2007).

The influence of the COMT Val 158Met polymorphism on controlled word retrieval should be revealed by generally faster reactions for the word/non-word decision of the lexical task for Met/Met carriers (high DA availability) in comparison to individuals with homozygote Val/Val (low DA availability) or the heterozygote Val/Met alleles (intermediate DA availability). If the same polymorphism also modulates semantic priming, homozygote Met/ Met carries should exhibit less masked and unmasked priming in comparison to individuals carrying at least one Val allel.

\section{MATERIALS AND METHODS PARTICIPANTS}

In total $N=104$ healthy Caucasian subjects, 90 women (age: $M=23.17, \mathrm{SD}=3.38$ ) and 14 men (age: $M=22.79, \mathrm{SD}=1.85$ ), whose mother tongue was German took part in the semantic priming experiments. The over-representation of female subjects was due to the fact that most of the participants were psychology students, a population with a majority of women. Participants were informed in detail about the purpose of the study and participated after they had signed the informed consent. Each subject obtained course credits for participation and could collect bonus points for a T-shirt labelled with their individual COMT genotype. The study was approved by the ethics committee of the German Psychological Association.

Mean age between men and women did not differ significantly $\left(F_{(1,102)}=0.17, p=0.682\right)$. There were no significant gender differences in any of the dependent variables/cognitive tasks. Therefore, a second factor 'sex' was not included into the MANOVA analyses besides COMT. Due to the skewed distribution of gender in our sample we were not able to test for sex by genotype interactions.

\section{EXPERIMENTAL PROCEDURE}

Lexical decision performance and semantic priming was tested with unmasked and masked versions of the priming paradigm. In both versions, word pairs (primes and targets) were sequentially presented in the center of a computer screen. Primes were always German words. One half of the critical prime-target pairs consisted of targets involving real German words, and the other half of the pairs were distractors with legally spelled pseudowords as targets. Subjects were instructed to decide as quickly and accurately as possible whether the target letter string was a real German word (lexical decision). Subjects indicated their decisions with a keypress for 'yes' or 'no' responses on a response box. The reaction times of the distractor trials were not analyzed. All participants were first presented with the masked priming paradigm and thereafter with the unmasked version. Reaction times were recorded from the onset of the target. The experiments were programmed and presented by means of the software Python 2.1 (www.python.org).

The masked priming paradigm was adapted from one of our previous experiments (e.g., Kiefer, 2002; Kiefer and Brendel, 2006) and consisted of 160 trials; 80 word-word pairs and 80 wordpseudoword pairs. Half of the word-word pairs consisted of 40 directly (hen-egg) and 40 non-related pairs (leaf-car). Targets of the related and non-related conditions were matched for word length and word frequency (Kiefer, 2002). Each trial started with a fixation cross $(750 \mathrm{~ms})$ followed by a mask consisting of nine randomly drawn letters that were presented for $100 \mathrm{~ms}$. Thereafter, the prime word was presented for $33 \mathrm{~ms}$. After the presentation of the prime another random letter mask was shown for $33 \mathrm{~ms}$ before the target stimulus, a German word or a pseudoword, appeared. Participants were instructed to make a lexical decision based upon the target stimulus. Participants were not informed on the presence of the prime behind the mask.

The unmasked version of the priming paradigm followed the procedure described in Kiefer et al. (2005) and consisted of 216 trials; 108 trials with a short SOA $(200 \mathrm{~ms})$ and 108 trials with a long SOA $(700 \mathrm{~ms})$. Within each SOA condition each subject responded to 54 word-word and 54 word-pseudoword pairs. These stimuli were divided into two lists. The assignment of a word list to the SOA conditions was counterbalanced across subjects. In order to vary the semantic relatedness between prime and target of the critical pairs 54 word-word trials of the two SOA conditions consisted of 18 non-related pairs (leaf-car), 18 indirectly related pairs (lemon-sweet), and 18 directly related pairs (hen-egg). Targets of the different semantic relatedness conditions were equated for word length and word frequency. Trials started with a fixation point for $700 \mathrm{~ms}$, followed by presentation of the prime for $200 \mathrm{~ms}$. In one condition, the target was immediately presented, thereafter resulting in an SOA of $200 \mathrm{~ms}$. In the other condition, the target was displayed after an inter-stimulus-interval of $500 \mathrm{~ms}$, yielding an SOA of $700 \mathrm{~ms}$. SOA was varied to distinguish between automatic and controlled priming processes. The target was displayed until the subject had responded. Trials of the two SOA conditions were blocked, and all subjects started with the SOA_200-ms condition followed by the SOA _ 700-ms condition. Within each block, trial order was initially randomized and thereafter kept constant. At the beginning of the priming experiments subjects performed 24 training trials, as well as 5 practice trials which were run immediately before each block.

After the experiments participants were totally debriefed on the experimental conditions, i.e. the existence of a prime and/or of the mask.

\section{GENETIC ANALYSES}

DNA was extracted from buccal cells to avoid a selective exclusion of subjects with blood and injection phobia. Automated purification of genomic DNA was conducted by means of the MagNA Pure ${ }^{\circledR}$ LC system using a commercial extraction kit (MagNA Pure LC DNA isolation kit; Roche Diagnostics, Mannheim, Germany). Genotyping of COMT Val158Met single nucleotide polymorphisms (SNPs) 
(rs4680) was performed by real time polymerase chain reaction (RT-PCR) using fluorescence melting curve detection analysis by means of the Light Cycler System (Roche Diagnostics, Mannheim, Germany). Details of the PCR protocols were described elsewhere (Reuter et al., 2005a, 2006). The primers and hybridization probes used (TIB MOLBIOL, Berlin, Germany) were as follows:

forward primer: 5'-GGGCCTACTGTGGCTACTCA-3'; reverse primer: $5^{\prime}$-GGCCCTTTTTCCAGGTCTG-3'; sensor hybridization probe: $5^{\prime}$-ATTTCGCTGGCATGAAGGA CAAG-fluorescein-3':

anchor hybridization probe: $5^{\prime}$-LCRed640-TGTGCATGCCTGAC CCGTTGTCA-phosphate-3'.

\section{RESULTS}

\section{GENOTYPE FREOUENCIES}

The COMT Val158met genotype frequencies were in HardyWeinberg-Equilibrium (Val/Val: $n=26$; Val/Met: $n=47$, Met/Met: $\left.n=31 ; \mathrm{Chi}^{2}=0.92, p>0.05\right)$.

\section{PRIMING EFFECTS INDEPENDENT OF THE COMT GENOTYPE}

Before testing the main hypothesis of our study, a possible effect of COMT Val158Met on semantic priming, we first conducted a manipulation check to test if semantic priming actually occurs. For this reason, to test for priming effects in the masked and unmasked priming conditions, separate repeated measures ANOVAs were calculated. The repeated measures factor had two levels in the masked priming condition (non-related, directly related) and three levels in the unmasked priming condition (non-related, indirectly related, directly related). For unmasked priming, separate repeated measures ANOVAs were run for short and for long SOAs. There were strong priming effects in all priming paradigms (see Table 1): masked priming $\left(F_{(1,103)}=52.92, p<0.00001\right)$, unmasked priming $\left(F_{(2,206)}=282.82, p<0.00001\right)$. In the two unmasked priming paradigms the order of reaction times was direct priming $<$ indirect priming < unrelated pairs. All possible post hoc contrasts (direct vs indirect, direct vs unrelated, indirect vs unrelated) were significant in the short and in the long SOA condition. The test statistics, means and SEMs are reported in Table 1.

\section{COMT AND PRIMING EFFECTS}

Again repeated measures ANOVAs (factor semantic relatedness) were calculated but this time with the additional independent factor COMT (Met/Met, Val/Met, Val/Val). In the masked priming paradigm, COMT did not significantly interact with semantic relatedness $\left(F_{(2,101)}=1.16, p=0.318\right)$. In order to specifically test the hypothesized influence of the COMT SNP on priming, we compared individuals with (Val+: Val/Val and Val/Met) and without a Val allele (Val-: Met/Met). In this analysis, the interaction between the factor Val+/Val- and semantic relatedness was also not significant $\left(F_{(1,102)}=2.30, p=0.13\right)$. However, as expected, individuals carrying at least one Val allele exhibited stronger masked priming than homozygote Met carriers. For the unmasked priming paradigm, the ANOVAs were conducted separately for short and long SOAs. In these analyses, all interactions including the factors COMT or Val+/Val- as between-subject factor and semantic relatedness as repeated measures factor were far from being significant (all $F<1, p>0.76$ ). Hence, we did not find a statistically reliable influence of the COMT polymorphism on masked and unmasked priming effects.

\section{COMT AND REACTION TIMES IN DIFFERENT PRIMING CONDITIONS}

A multivariate analysis of variance with the independent factor COMT and the mean reaction times in all priming conditions as dependent variables was calculated. The COMT genotype was significantly associated with all dependent variables in the semantic priming tasks, no matter if in the masked or unmasked priming task, if short or long SOAs were used, or if the prime target associations were direct, indirect or unrelated. The results of the MANOVA including means and SEMs are presented in Table 2.

\section{COMT AND ACCURACY}

COMT Val158Met was not related to differences in error rates irrespective of priming condition. Therefore, differences in reaction times between different genotype groups could not be accounted for by genotype dependent differences in accuracy. It has to be mentioned, that only correct trial were included in the analyses of priming effects (see above).

\section{DISCUSSION}

The present study assessed the modulatory influence of the COMT Val158met polymorphism on lexical decision latencies within masked and unmasked versions of the semantic priming paradigm. We found a strong effect of the COMT genotype on overall lexical decision latencies, putatively due to the relatively higher functionality of the executive control system in homozygote Met carriers. In contrast to these strong effects on lexical decisions, semantic

Table 1 | Priming effects.

\begin{tabular}{|c|c|c|c|c|c|}
\hline & Direct priming $(A)$ & Indirect priming (B) & Unrelated prime-target (C) & MANOVA & Post hoc tests Bonferroni \\
\hline \multirow[t]{2}{*}{ Unmasked priming short SOA } & $M=573.20$ & $M=606.44$ & $M=638.28$ & $F=149.92$ & $A<B$ \\
\hline & $\mathrm{SEM}=7.90$ & $\mathrm{SEM}=8.39$ & $\mathrm{SEM}=8.34$ & $p<0.00001$ & $A<C$ \\
\hline \multirow[t]{2}{*}{ Unmasked priming long SOA } & $M=549.97$ & $M=588.37$ & $M=614.71$ & $F=153.73$ & $A<B$ \\
\hline & $\mathrm{SEM}=6.90$ & $\mathrm{SEM}=6.70$ & $\mathrm{SEM}=7.02$ & $p<0.00001$ & $A<C$ \\
\hline & $\mathrm{SEM}=7.04$ & - & $\mathrm{SEM}=7.66$ & $p \leq 0.00001$ & \\
\hline & & & & eta $^{2}=0.339$ & \\
\hline
\end{tabular}


Table 2 | Association between lexical decision latencies in the semantic priming tasks and the COMT Val158Met polymorphism.

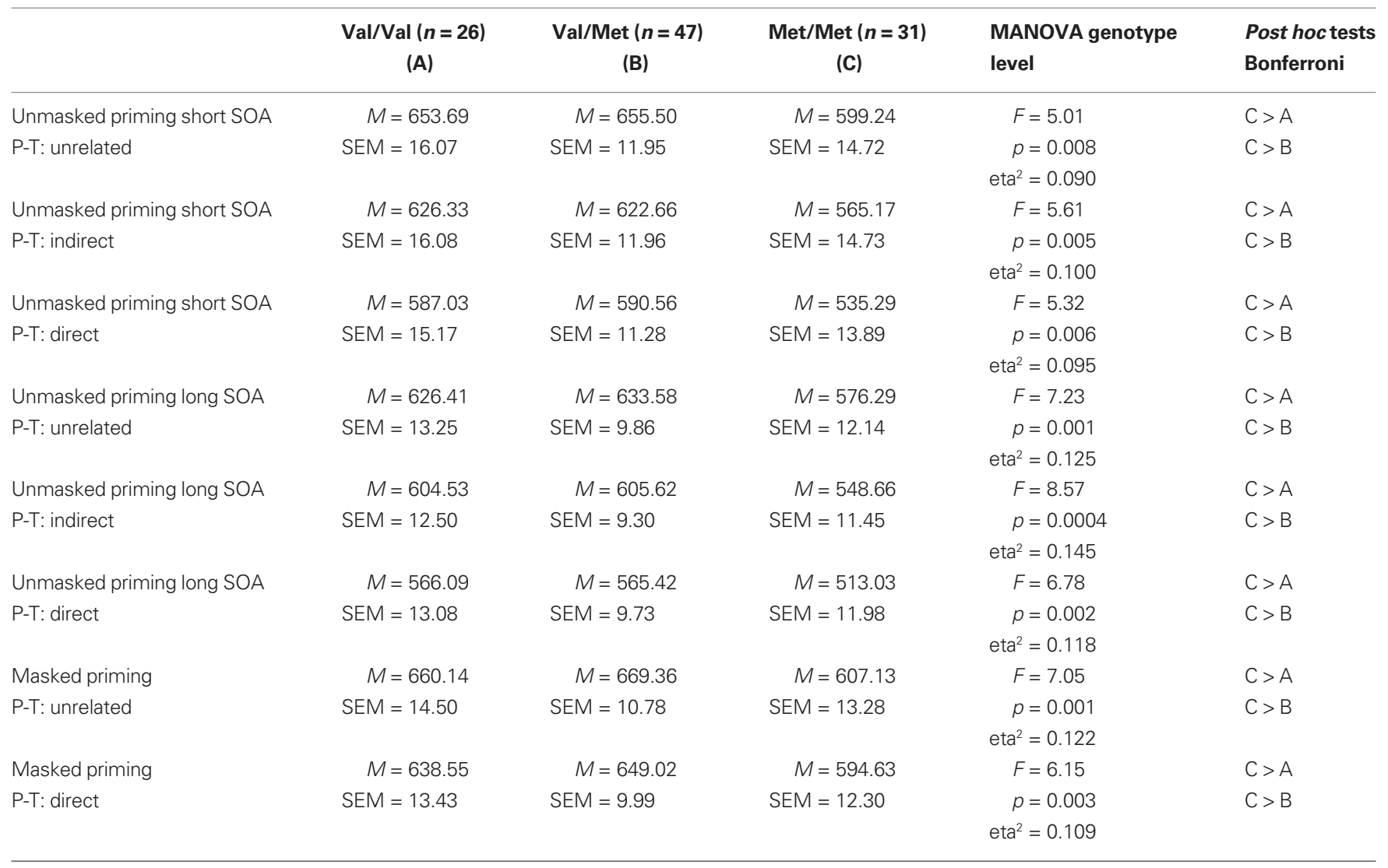

Means (M) and standard errors of means (SEM) for the priming tasks were reported in milliseconds.

priming was not reliably modulated by the COMT genotype. However, as expected, individuals carrying at least one Val allele exhibited stronger masked priming than homozygote Met carriers. However, given the weak tendency of the effect and the number of tests calculated we must deny an effect of COMT on semantic priming based on the present data. Due to earlier findings showing that typically slower RTs allow for larger difference scores (e.g. Kiefer et al., 2005) - the Val+ group had overall slower RTs - the priming effect is more pronounced in this group. In future work using a more heterogeneous sample with a higher variability in reaction times a possible effect of COMT on semantic priming is more likely to be identified.

Our results suggest that individual differences in activity of the prefrontal DA system induced by the COMT Val158met polymorphism largely modulate executive functions (as indicated by the fact that RTs for Met/Met are substantially faster than for Val+) and to less extent automatic semantic processing [the size of three different semantic priming effects (unmasked 'automatic', unmasked 'conscious', and masked) are the same across Met/Met, Met/Val, and Val/Val]. Therefore, individual differences in activity of the prefrontal DA system induced by the COMT Val158met polymorphism largely modulate executive functions while having no significant effect on automatic semantic processing.

Influential studies in the cognitive neurosciences have demonstrated that the PFC is elementary for executive control processes including working memory, conflict resolution, set shifting and semantic processing (e.g. Kiefer et al., 1998; D’Esposito et al., 2000; Duncan and Owen, 2000; Liu et al., 2008; Manenti et al., 2008; Reuter et al., 2008; Volle et al., 2008; Specht et al., 2009). Executive control is hypothesized as the common underlying process of these different cognitive tasks (Duncan and Owen, 2000). Support for this hypothesis comes from molecular genetics demonstrating that a functional single nucleotide polymorphism (SNP) of the COMT gene is associated with heterogeneous executive tasks like the WCST or working memory as assessed by the n-back task (e.g. Egan et al., 2001; Goldberg and Weinberger, 2004). These findings suggest that the different tasks share some common variance. Probably the variance accounted for by the COMT Val158Met SNP represents in part the functionality of the prefrontal executive control system. Positive findings in genetic association studies are not only an indicator for heritability, at least to a certain extent, they are not an end in itself for ambitious scientists, but they provide us information on the neurochemical underpinnings of a certain phenotype. In the case of the COMT gene we might conclude from this positive association studies that the dopaminergic system is involved in the processing of executive control functions related to the PFC. Besides pharmacolocical and clinical neuropsychology research, the molecular genetic approach constitutes an elegant approach to trace down the biochemical basis of cognitive functions. Moreover, the genetic approach overcomes risks and/or 
shortcomings of the other two methods: The administration of drugs to healthy subjects raises ethical issues and patients can rarely be investigated in an unmedicated status (Serretti et al., 2008). The relationship between COMT Val158Met and cognitive functioning is one of the most investigated gene-phenotype association with a majority of studies reporting an allele load effect for the Met allele or at least superior performance in carriers of the Met allele (Met/Met an Val/Met genotypes). Because the Met allele is related to a tremendous reduction in the degradation of dopamine in the PFC it is hypothesized that high cognitive performance is related to high prefrontal dopamine levels. However, pharmacogenetic studies have demonstrated that the linear relationship between prefrontal dopamine availability and performance switches to an inverted U-shaped one if a certain dopamine concentration is exceeded (Mattay et al., 2003). Although semantic priming is one of the most prominent paradigms in cognitive science it has not been tried to extrapolate positive associations between COMT and executive control to linguistic processing and semantic priming. We did this in a sample of $N=104$ healthy students. Investigations in students have often been criticized to be limited to this special population and to be without any significance for the general population. However, we state that a student sample is also of advantage. All students are relatively homogeneous with respect to intelligence - at least the variance is far more restricted than in a population-based study. Restricted variance also means conservative testing, i.e. it is more difficult to obtain significant results.

Our study shows that semantic priming effects were not influenced by the COMT SNP. Numerically but not statistically significant we observed reduced masked priming effect in homozygote carriers of the Met allele as could be expected from earlier pharmacological studies (Kischka et al., 1996). However, the COMT SNP did not influence unmasked priming and in particular indirect priming at all. The entire lack of an effect of COMT on unmasked priming contrasts with earlier results from a pharmacological study in healthy volunteers which demonstrated reduced unmasked indirect priming after administration of L-dopa, a precursor of dopamine (Kischka et al., 1996). There are several explanations to account for this discrepancy: First, it is possible that the influence of the COMT SNP on PFC dopamine availability is much weaker in comparison to the administration of L-dopa during a pharmacological challenge. Second, the COMT SNP may not be the only genetic polymorphism affecting DA activity. It is conceivable that semantic priming is more strongly influenced by other genetic polymorphism than COMT. This issue, however, has to be addressed in future studies.

In our study, the COMT SNP had a resounding COMT effect on lexical decision latencies irrespective of the precise priming task. Between 9 and $14.5 \%$ of the variance in the reaction times of the

\section{REFERENCES}

Balota, D. A., and Lorch, R. F., Jr. (1986). Depth of automatic spreading activation: mediated priming effects in pronunciation but not in lexical decision. J. Exp. Psychol. Learn. Mem. Cogn. 12, 336-345.
Barnett, J. H., Jones, P. B., Robbins, T. W., and Müller,U. (2007). Effects of the catechol-O-methyltransferase Val158Met polymorphism on executive function: a meta-analysis of the Wisconsin Card Sort Test in schizophrenia and healthy controls. Mol. Psychiatry 2, 502-509.

lexical decision task can be explained by a single base pair exchange on the COMT gene. In terms of a molecular genetic study this is a very strong effect, however, there is a long way to go until all SNPs relevant for the performance in lexical decision tasks are identified.

The COMT effect reported in this study appears to suggest a general superiority of carriers of the Met/Met genotype in visual word recognition within a lexical decision task. In a lexical decision task, lexical, phonological and semantic word information has to be retrieved in a strategic fashion (Neely, 1991). In line with this view, the lexical decision task recruits PFC regions with enhanced PFC activity when the demands on controlled word retrieval were high. For instance, PFC activity increased as a function of the similarity between words and pseudowords and depended on word frequency (Liu et al., 2004; Edwards et al., 2005). We assume that high DA activity in carriers of the Met/Met genotype is associated with a superior functionality of PFC circuits in comparison to carriers of at least one Val allele.

The dissociation between positive findings in the lexical decision task and negative findings with respect to the semantic priming are in line with functional MRI studies demonstrating that controlled semantic priming relies more on PFC regions than automatic semantic priming (Gold et al., 2006). Presumably, in our study, even the priming conditions with a long SOA between prime and target did not sufficiently induce controlled priming mechanisms due to the presence of indirectly related prime-target pairings, which reduce the likelihood of a successful prediction of the subsequent target upon prime presentation (Spitzer et al., 1993). This considerably prevents the application of expectancy mechanisms as a cognitive basis for priming (e.g., Neely, 1991).

In a previous study we investigated the effect of the COMT SNP on simple motoric reaction times (Reuter et al., 2005b). Due to the absence of a positive association in that earlier study, we can conclude that the present findings are not the result of simple motoric reaction time differences but specifically reflect processing supporting lexical decisions carried out in PFC. The present study supports and extends findings from previous studies relating the Met allele or the Met/Met genotype to high performance in tasks where executive control is involved (for an overview see Goldberg and Weinberger, 2004). The presently observed effect on lexical decisions demonstrates that the COMT gene also influences cognitive tasks that go beyond classical executive control tasks. Hence, the COMT gene has a broad impact on cognitive functioning and even influences visual word recognition during lexical decision-making.

\section{ACKNOWLEDGEMENT}

We would like to thank Dr. Luke Smilllie, Department of Psychology,Goldsmith Institute, London, for his help and intellectual input.

Bertolino, A., Blasi, G., Latorre, V., Rubino, V., Rampino, A., Sinibaldi, L., Caforio, G., Petruzzella, V., Pizzuti, A., Scarabino, T., Nardini, M. Weinberger, D. R., and Dallapiccola, B. (2006). Additive effects of genetic variation in dopamine regulating genes on working memory cortical activity in human brain. J. Neurosci. 26, 3918-3922.

Callaway, E., 3rd. (1970). Schizophrenia and interference. An analogy with a malfunctioning computer. Arch. Gen Psychiatry 22, 193-208. 
de Groot, A. M. B. (1983). The range of automatic spreading activation in word priming. J. Verb. Learn Verb. Behav. 22, 417-436.

D’Esposito, M., Postle, B. R., and Rypma, B. (2000). Prefrontal cortical contributions to working memory: evidence from event-related fMRI studies. Exp. Brain Res. 133, 3-11.

Dolan, R. J., Fletcher, P., Frith, C. D., Friston, K. J., Frackowiak, R. S., and Grasby, P. M. (1995). Dopaminergic modulation of impaired cognitive activation in the anterior cingulate cortex in schizophrenia. Nature 378, 180-182.

Duncan, J. (2001). An adaptive coding model of neural function in prefrontal cortex. Nat. Rev. Neurosci. 2, 819-829.

Duncan, J., and Owen, A. M. (2000). Common regions of the human frontal lobe recruited by diverse cognitive demands. Trends Neurosci. 23, 475-482.

Edwards, J. D., Pexman, P. M., Goodyear, B. G., and Chambers, C. G. (2005). An fMRI investigation of strategies for word recognition. Cogn. Brain Res. 24, 648-662.

Egan, M. F., Goldberg, T. E., Kolachana, B.S., Callicott, J. H., Mazzanti, C. M., Straub, R. E., Goldman, D., and Weinberger, D. R. (2001). Effect of COMT Val108/158 Met genotype on frontal lobe function and risk for schizophrenia. Proc. Natl. Acad. Sci. U.S.A. 98, 6917-6922.

Faw, B. (2003). Pre-frontal executive committee for perception, working memory, attention, long-term memory, motor control, and thinking: a tutorial review. Conscious. Cogn. 12, 83-139.

Gold, B. T., Balota, D. A., Jones, S. J., Powell, D. K., Smith, C. D., and Andersen, A. H. (2006). Dissociation of automatic and strategic lexicalsemantics: functional magnetic resonance imaging evidence for differing roles of multiple frontotemporal regions. J. Neurosci. 26, 6523-6532.

Goldberg, T. E., Egan, M. F., Gscheidle, T., Coppola, R., Weickert, T., Kolachana B. S., Goldman, D., and Weinberger, D. R. (2003). Executive subprocesses in working memory: relationship to catecholO-methyltransferase Val158Met genotype and schizophrenia. Arch. Gen. Psychiatry 60, 889-896.

Goldberg, T. E., and Weinberger, D. R. (2004). Genes and the parsing of cognitive processes. Trends Cogn. Sci. 8, 325-335.

Kiefer, M. (2002). The N400 is modulated by unconsciously perceived masked words: further evidence for a spreading activation account of N400 priming effects. Cogn. Brain Res. 13, 27-39.

Kiefer, M. (2007). Top-down modulation of unconscious 'automatic' processes: a gating framework. Adv. Cogn. Psych. 3, 289-306.

Kiefer, M., Ahlegian, M., and Spitzer, M. (2005). Working memory capacity, indirect semantic priming, and stroop interference: pattern of interindividual prefrontal performance differences in healthy volunteers. Neuropsychology 19, 332-344.

Kiefer, M., and Brendel, D. (2006). Attentional modulation of unconscious 'automatic' processes: evidence from event-related potentials in a masked priming paradigm. J. Cogn. Neurosci. 18, 184-198.

Kiefer, M., Marzinzik, F., Weisbrod, M., Scherg, M., and Spitzer, M. (1998). The time course of brain activations during response inhibition: evidence from event-related potentials in a Go/Nogo task. Neuroreport 9, 765-770.

Kiefer, M., Schuch, S., Schenck, W., and Fiedler, K. (2007). Mood states modulate activity in semantic brain areas during emotional word encoding. Cereb. Cortex 17, 1516-1530.

Kiefer, M., Sim, E.-J., Herrnberger, B., Grothe, J., and Hoenig, K. (2008). The sound of concepts: four markers for a link between auditory and conceptual brain systems. J. Neurosci. 28, 12224-12230.

Kiefer, M., Weisbrod, M., Kern, I., Maier, S., and Spitzer, M. (1998). Right hemisphere activation during indirect semantic priming: evidence from event-related potentials. Brain Lang. 64, 377-408.

Kischka, U., Kammer, T., Weisbrod, M., Maier, S., Thimm, M., and Spitzer, M. (1996). Dopaminergic modulation of semantic network activation. Neuropsychologia 34, 1107-1113.

Lachman, H. M., Papolos, D. F., Saito, T., Yu, Y. M., Szumlanski, C. L., and Weinshilboum, R. M. (1996). Human catechol-O-methyltransferase pharmacogenetics: description of a functional polymorphism and its potential application to neuropsychiatric disorders. Pharmacogenetics 6, 243-250.

Liu, H.L., Liao, W. T., Fang, S.Y., Chu, T. C., and Tan, L. H. (2004). Correlation between temporal response of fMRI and fast reaction time in a language task. Magn. Reson. Med. 22, 451-455.

Liu, J., Bai, J., and Zhang, D. (2008). Cognitive control explored by linear modelling behaviour and fMRI data during Stroop tasks. Physiol. Meas. 29, 703-710.

Maher, B. A., Manschreck, T. C., Redmond, D., and Beaudette, S. (1996). Length of illness and the gradient from positive to negative semantic priming in schizophrenic patients. Schizophr. Res. 22, 127-132.

Manenti, R., Cappa, S. F., Rossini, P. M. and Miniussi, C. (2008). The role of the prefrontal cortex in sentence comprehension: an rTMS study. Cortex 44 337-344.

Manschreck, T. C., Maher, B. A., Milavetz,J.J., Ames, D., Weisstein, C. C., and Schneyder, M. L. (1988). Semantic priming in thought disordered schizophrenic patients. Schizophr. Res. 1, 61-66.

Mattay, V. S., Goldberg, T. E., Fera, F., Hariri, A. R., Tessitore, A., Egan, M. F., Kolachana, B., Callicottm, J. H., and Weinberger, D. R. (2003). Catechol O-methyltransferase val158-met genotype and individual variation in the brain response to amphetamine. Proc. Natl. Acad. Sci. U.S.A. 100 6186-6191.

Monchi, O., Petrides, M., Petre, V., Worsley, K., and Dagher, A. (2001). Wisconsin Card Sorting revisited: distinct neural circuits participating in different stages of the task identified by event-related functional magnetic resonance imaging. J. Neurosci. 21, 7733-7741.

Neely, J.H. (1977). Semantic priming and retrieval from lexical memory: roles of inhibitionless spreading activation and limited-capacity attention. J. Exp. Psychol. 106, 226-254.

Neely, J. H. (1991). Semantic priming effects in visual word recognition: a selective review of current findings and theories. In Basic Progresses in Reading - Visual Word Recognition, D. Besner and G. W. Humphreys, eds (Hillsdale, NJ, Lawrence Erlbaum Associates), pp. 264-333.

Poldrack, R.A., Wagner,A.D., Prull, M. W. Desmond, J. E., Glover, G. H., and Gabrieli, J. D. (1999). Functional specialization for semantic and phonological processing in the left inferior prefrontal cortex. Neuroimage 10 15-35.

Posner, M. I., and DiGirolamo, G. J. (1998). Executive attention: conflict, target detection and cognitive control. In The Attentive Brain, R. Parasuraman, ed. (Cambridge, MA, MIT Press), pp. 401-423.

Posner, M. I., and Snyder, C. R. R. (1975). Facilitation and inhibition in the processing of signals. In Attention and Performance,Vol. 5, P. M. Rabbitt and S. Dornic, eds (San Diego, CA, Academic Press).

Reuter, M., Esslinger, C., Montag, C., Lis, S., Gallhofer, B., and Kirsch, P. (2008). A functional variant of the tryptophan hydroxylase 2 gene impacts working memory: a genetic imaging study. Biol. Psychol. 79, 111-117.
Reuter, M., Küpper, Y., Schmitz, A., Breuer, J. P., Wend, U., and Hennig, J. (2005a). Detection of new single nucleotide polymorphisms by means of Real Time PCR. J. Genet. 84, 341-345.

Reuter, M., Peters, K., Schroeter, K., Koebke, W., Lenardon, D., Bloch, B., and Hennig, J. (2005b). The influence of the dopaminergic system on cognitive functioning: a molecular genetic approach. Behav. Brain. Res. 164, 93-99.

Reuter, M., Schmitz, A., Corr, P., and Hennig, J. (2006). Molecular genetics support Gray's personality theory: The interaction of COMT and DRD2 polymorphisms predicts the behavioral approach system. Int. J. Neuropsychopharmacol. 9, 155-166.

Serretti, A., Kato, M., and Kennedy, J. L. (2008). Pharmacogenetic studies in depression: a proposal for methodologic guidelines. Pharmacogenomics 8, 90-100.

Specht, K., Lie, C. H., Shah, N. J., and Fink, G. R. (2009). Disentangling the prefrontal network for rule selection by means of a non-verbal variant of the Wisconsin Card Sorting Test. Hum. Brain Mapp. 30, 1734-1743.

Spitzer, M., Braun, U., Hermle, L., and Maier, S. (1993). Associative semantic network dysfunction in thoughtdisordered schizophrenic patients: direct evidence from indirect semantic priming. Biol. Psychiatry 34, 864-877.

Spitzer, M., Weisker, I., Maier, S., Hermle, L., and Maher, B. A. (1994). Semantic and phonological priming in schizophrenia. J. Abnorm. Psychol. 103, 485-494.

Thompson-Schill, S. L. (2003). Neuroimaging studies of semantic memory: inferring "how" from "where". Neuropsychologia 41, 280-292.

Tunbridge, E. M., Harrison, P. J., and Weinberger, D. R. (2006). Catecholo-methyltransferase, cognition, and psychosis: Val158Met and beyond. Biol. Psychiatry 60, 141-151.

Vijayraghavan, S., Wang, M., Birnbaum, S. G., Williams, G. V., and Arnsten, A. F. (2007). Inverted-U dopamine D1 receptor actions on prefrontal neurons engaged in working memory. Nat. Neurosci. 10, 376-384.

Volle, E., Kinkingnéhun, S., Pochon, J. B., Mondon, K., Thiebaut de Schotten, M., Seassau, M., Duffau, H., Samson, Y., Dubois, B., and Levy, R. (2008). The functional architecture of the left posterior and lateral prefrontal cortex in humans. Cereb. Cortex 18, 2460-2469.

Wagner, A. D., Paré-Blagoev, E. J., Clark, J., and Poldrack, R.A. (2001). Recovering 
meaning: left prefrontal cortex guides controlled semantic retrieval. Neuron 31, 329-338.

Weisbrod, M., Maier, S., Harig, S., Himmelsbach, U., and Spitzer, M. (1998). Lateralized semantic and indirect semantic priming effects in people with schizophrenia. Br. J. Psychiatry 172, 142-146.

Winterer, G., and Goldman, D. (2003). Genetics of human prefrontal func- tion. Brain Res. Brain Res. Rev. 43, 134-163.

Winterer, G., and Weinberger, D. R. (2004). Genes, dopamine and cortical signal-to-noise ratio in schizophrenia. Trends Neurosci. 27, 683-690.

Conflict of Interest Statement: The authors declare that the research was conducted in the absence of any commercial or financial relationships that could be construed as a potential conflict of interest.

Received: 03 April 2009; paper pending published: 20 April 2009; accepted: 31 July 2009; published online: 24 August 2009.

Citation: Reuter M, Montag C, Peters K, Kocher A and Kiefer M (2009) The modulatory influence of the functional COMT Val158Met polymorphism on lexical decisions and semantic priming.
Front. Hum. Neurosci. 3:20. doi 10.3389/neuro.09.020.2009

Copyright (c) 2009 Reuter, Montag, Peters, Kocher and Kiefer. This is an open-access article subject to an exclusive license agreement between the authors and the Frontiers Research Foundation, which permits unrestricted use, distribution, and reproduction in any medium, provided the original authors and source are credited. 